Scientific Visualization, 2020, volume 12, number 4, pages 133 - 142, DOI: 10.26583/sv.12.4.12

\title{
Visualization of Graph-based representations for analyzing related multidimensional objects
}

\author{
M.S. Ulizko1,A,B, E.V. Antonov',A,B, A.A. Artamonov 3,B, R.R. Tukumbetova4,A,B \\ A Plekhanov Russian University of Economics \\ B National Research Nuclear University MEPhI (Moscow Engineering Physics Institute) \\ ${ }^{1}$ ORCID: oooo-0003-26o8-8330, mulizko@kaf65.ru \\ 2 ORCID: oooo-0003-1498-9131, eantonov@kaf65.ru \\ 3 ORCID: 0000-0002-9140-5526, aartamonov@kaf65.ru \\ 4 ORCID: 000o-0002-1976-1390, rrtukumbetova@kaf65.ru
}

\begin{abstract}
The paper considers the task of analyzing complex interconnected objects using graphs. The subject of the research is such multidimensional object as a "politician". The paper presents the main methods of visualizing multidimensional data and the choice of data analysis is justified using graphs. An algorithm that enables to automate data collection on politicians was developed. One of the main methods of data collecting on the Internet is web scraping. Web scraping software may access the World Wide Web directly using the HTTP, or through a web browser. While web scraping can be done manually by a software user, the term typically refers to automated processes implemented using a software agent.

The process of transition from an object of the "personality" type to its graph representation with various types of nodes and edges is described. The use of graphs enables to see various types of relationships, including mediate. The features of the used Gephi software for data analysis are presented.

This methodology enables to change the attitude towards the analysis of multidimensional objects.
\end{abstract}

Keywords: Web development, graph, visualization, politicians.

\section{Data collection and processing}

In the modern world there is a large amount of information that can be represented as objects and the relationship between them. For example, objects can be individuals, whose relationship is described by a certain characteristic. In this case, analysis can be conducted in various ways: elastic map method, cluster analysis, graphs plotting, etc. The paper presents graph representation of an individual. The reason for choosing this method is specified below [1].

Political forces often play an important role for the development of scientific and social activities within individual states and the world community. Decisions made by political forces may depend on the individual characteristics of the person (gender, age, fraction) and the relationship between them.

Information about political figures is also posted on the Internet. It enables to obtain data in sufficient quantities. It is unreasonable to collect data on politicians manually, since there is a lot of information. Therefore, algorithms are being developed that enable to collect data from Internet information sources.

To build graphs for political leaders, it is necessary to determine the nodes of the graphs and describe the relationship between them.

Graph plotting is an unnatural process for the computer. With existing software it is rarely possible to construct large graphs or have the ability to interact. However, with the correct 
construction, graphs enable a qualitative data analysis and identify both explicit and hidden relationships [2-4].

\section{Methodology}

\subsection{Related works}

The easiest way for a person to perceive data is as $2 \mathrm{D}$ objects, but often data has a larger dimension (number of fields / attributes can reach hundreds of orders). This raises two main questions:

- How to bring the data to the form suitable for visualization?

- How to represent multidimensional objects in a space of lower dimensions?

The fact is that it is good to have a two-dimensional picture in front of you, which would reflect the data patterns: cluster structure, the presence of dependencies between objects, etc.

The problem of displaying multidimensional data in a 2D measurement is currently well studied - various approaches can be used for visualization $[5,6]$ :

- $\quad$ elastic map,

- cluster analysis,

- neural network,

- data analysis on graphs,

- others.

The choice of how to visualize data is paramount and often depends on the data itself.

If all attributes are numeric, boolean, or convertible, the first three methods can be used. In this case, it is necessary to determine the choice of the projection plane (for example, the plane of the first two main components) and the principle of projection (some extreme functional).

If the researcher is more interested not in data, but in the relationships, data analysis on graphs is more often used.

The initial data on politicians have the following characteristics:

- most of the fields are of the text data type,

- $\quad$ fields can be nested and / or have a list view (consists of several elements of the same type).

- $\quad$ objects can be linked based on several fields - all links must be identified.

According to the peculiarities of the data and the formulation of the problem (study of the relationships between objects), we consider the data using graphs.

At the same time, we divide the process of collecting and analyzing data into the following components:

- Data collection (Extract)

- $\quad$ Data processing (Transform)

- $\quad$ Loading data into some storage (Load)

- Visualization and analysis.

\subsection{Collecting data about politicians on the Internet}

One of the main methods of data collecting on the Internet is web scraping. The general principle of web scraping can be represented as follows: the program code sends a request to the target source and receives a response in the form of HTML code, after which it searches for the required information using the XPath query language.

Requests are most often generated using the HTTP protocol; they use the GET request method to receive data.

The response is an object that is predisposed to receive any data. In data processing, an HTML document is extracted from the response, after which a search is performed, and the result is converted to the required format. This process is called parsing. 
Web scraping can be done in two ways:

- $\quad$ development of software;

- $\quad$ use of third-party software (including API).

If it is necessary to use several information sources at the same time for data extraction, the second method is usually not used. Automate data collection during the development of software can be through the use of agent technologies (software agents) [7, 8]. Software agent is a computer program that acts on behalf of a user on demand or according to a schedule. Additional software packages can be also used to interact with the browser. When developing software to solve this problem, the programming languages Python and JavaScript are most often used.

Information about politicians is available on various Internet sources. As an exam- ple, information from such sources as VoteSmart and Govtrack is considered [7, 8]. They have a complex structure, so it is impossible to use third-party software. For the development, we used the Python programming language and software packages of the language, such as selenium, lxml.

The data collection algorithm is divided into three subtasks:

- $\quad$ GET request to an information source;

- $\quad$ extracting data from the response body (HTML document) using Xpath;

- $\quad$ saving the received data with the possibility of further visualization.

Thus, the algorithm is as follows (see Fig. 1).

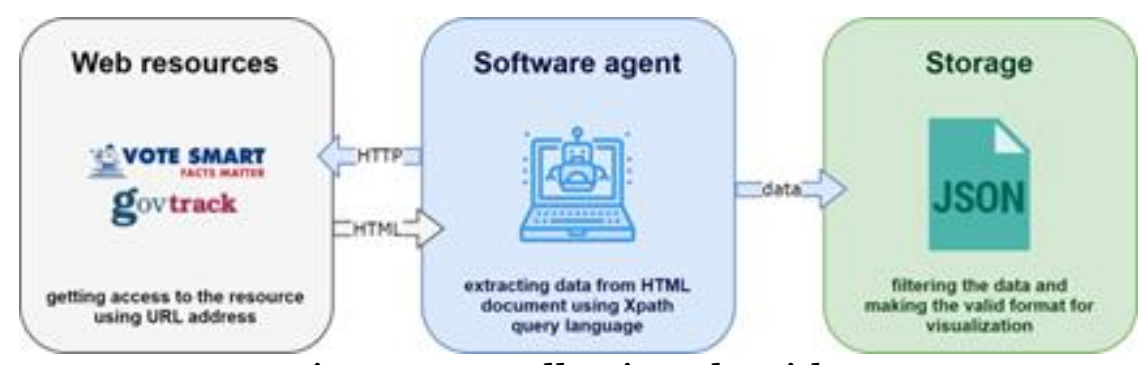

Fig. 1. Data collection algorithm

JSON is selected as the data presentation format. The choice is due to the complex nested structure of the initial data, which is difficult to process when stored in CSV format. Meanwhile, the XML format is redundant [11].

\subsection{Graph representation of personality}

For the analysis of interrelated objects graphical representation of the data are used. A graph consists of vertices (nodes) and edges that form a connection between vertices. In order to determine the nodes and edges, we select the characteristics of the person obtained during data collection:
- name
- party
- district
- ratings
- bills
- religion
- education
- political experience
- current legislative committees
- former committees
- professional experience
- other organizations 
- additional information (awards, favorite quotes, etc.)

The figure shows an example of the data collected by a politician in JSON (see Fig. 2).

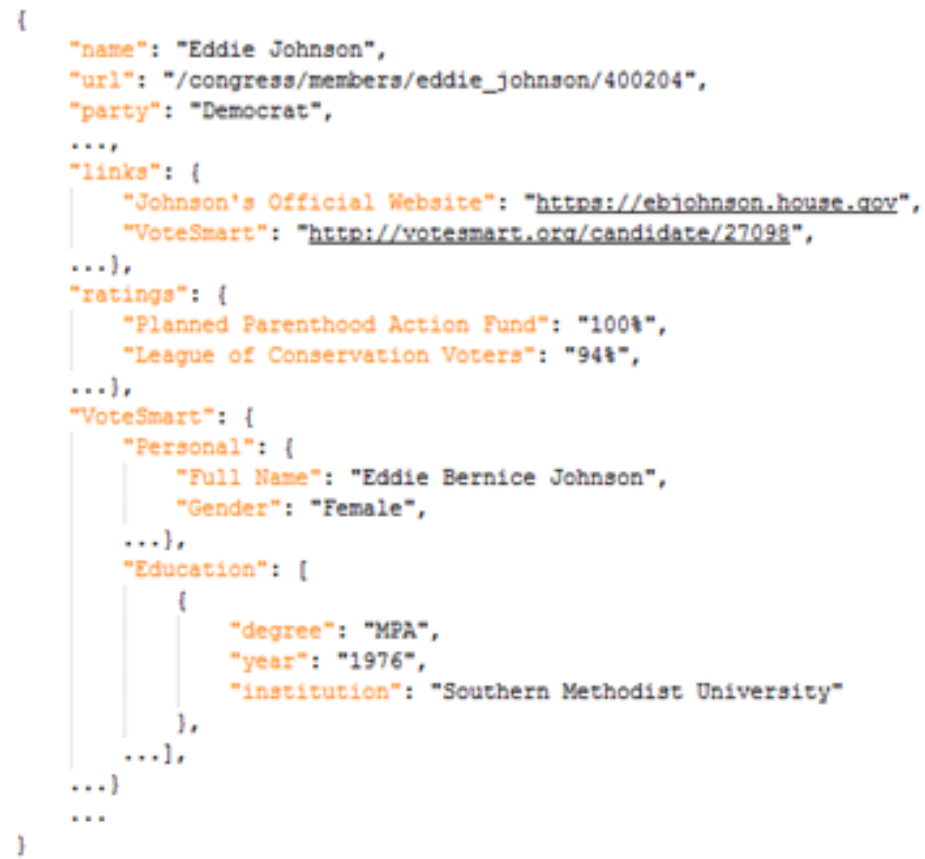

Fig. 2. Example of politician data

Due to the fact that a person has many properties, when constructing a graph for politicians, two approaches arise:

- $\quad$ the node of the graph is the person, an edge - some of the properties;

- $\quad$ several types of nodes are distinguished from the properties of the person, in this case the edges reflect the correspondence of nodes in the initial data.

In the first approach, for the trivial case, nodes can be connected by an edge of the same type (for example, by belonging to the same party). In this case, between the nodes either there is or there is no a node. The approach can be expanded by introducing edges for each of the considered properties and introducing markers (color, weight) to distinguish between the edges. For clarity, there should be no more than 5 different types of edges.

For the second approach, the nodes are unique values of characteristics; edges are the relationship between these characteristics in the initial data. To construct such a graph, it is required to consider in what form the information is presented.

The initial data are separate JSON files, which contain information about representatives of some of the largest US states: Texas, California and Florida. To construct a graph it is necessary to explicitly distinguish nodes and edges. We divide the nodes into categories, while all nodes can be connected by edges only through the node identity.

In the typical case a graph is defined by an adjacency matrix, but other methods are often used. In particular, if there are many "zeros" in the adjacency matrix, which indi- cate that there is no connection between nodes, the graph is specified with a list. The list consists of entries of the following form (1):

$$
\begin{gathered}
\left\langle v_{i}, v_{j}, w_{i, j}\right\rangle \text {, where } \\
v_{i} \text { - starting node, } v_{j}-\text { end node, } w_{i, j}-\text { edge weight }
\end{gathered}
$$

In this case the graph will be undirected. The edge only indicates the connection between the nodes, not the nature of the connection. We distinguish the following categories of nodes:
- Person
- Educational institution
- Religion 
- Membership in a civil organization

- Party

Because of the initial data representation one of the nodes of each edge will be a 'person' node.

The graph may be weighted and unweighted. Weight can be introduced if there is a one-tomany relationship between objects. In particular, such a relationship is formed in such node as 'person - university' and 'person - membership in a civil organization'. In this case, the weight of the edge will be specified and show the relative share of the university / organization for a particular person. Mathematically this is represented as the following formula (2):

$$
\sum_{i=1}^{k_{j}} w_{j, i}=1 \text { for } \forall j \text {, where }
$$

\section{$j-{ }^{I}$ person $^{I}$ node, $i-$ instiutions connected with node $\mathrm{j}$}

To get a graph structure (the Transform step in the previously described data collection and analysis scheme), two factors should be taken into account:

- $\quad$ it is necessary to manually (algorithmically) select nodes of various types and relationships between them,

- it is necessary to take into account that nodes can be repeated in the initial data. There are two main approaches to take into account the second factor:

- maintenance of uniqueness of data when creating / adding - if at some stage a node is encountered that is already in the graph, a new node is not created, but only its relationships are added,

- maintenance of uniqueness of data due to queries - if, when creating / adding, a node is encountered that is already in the graph, then this node is first created, but then a MERGE-type query occurs, which combines identical nodes with each other.

The Python programming language is used to obtain the graph structure. The developed algorithm converts the initial JSON data into nodes and edges, which are stored in separate CSV files.

\subsection{Visualization and analysis}

There is no unified tool for constructing graphs. Some solutions can build graphs limited by the number of nodes, while others do not visually display data $[12,13]$. One commonly used application is Gephi [14]. Gephi is a free, open-source data visualization product. It is a user interactive software and can be applied for constructing complex systems.

When constructing a graph, we hypothesize that connected nodes are close to each other, and unconnected or opposite nodes are located at a great distance from each other.

To test this hypothesis, we choose the Force Atlas layout algorithm, having previously colored the nodes depending on the type of characteristic. The algorithm is based on minimizing 'energy' (the nodes are iteratively attracted or repelled from each other in the visualization space, depending on their relative position and the presence of relationships). Thus, the algorithm will be sensitive to the chosen hypothesis.

We build a graph with the initial data by customizing the display of the output. The graph takes the following form (see Fig. 3) and contains 4601 nodes and 5993 edges. 


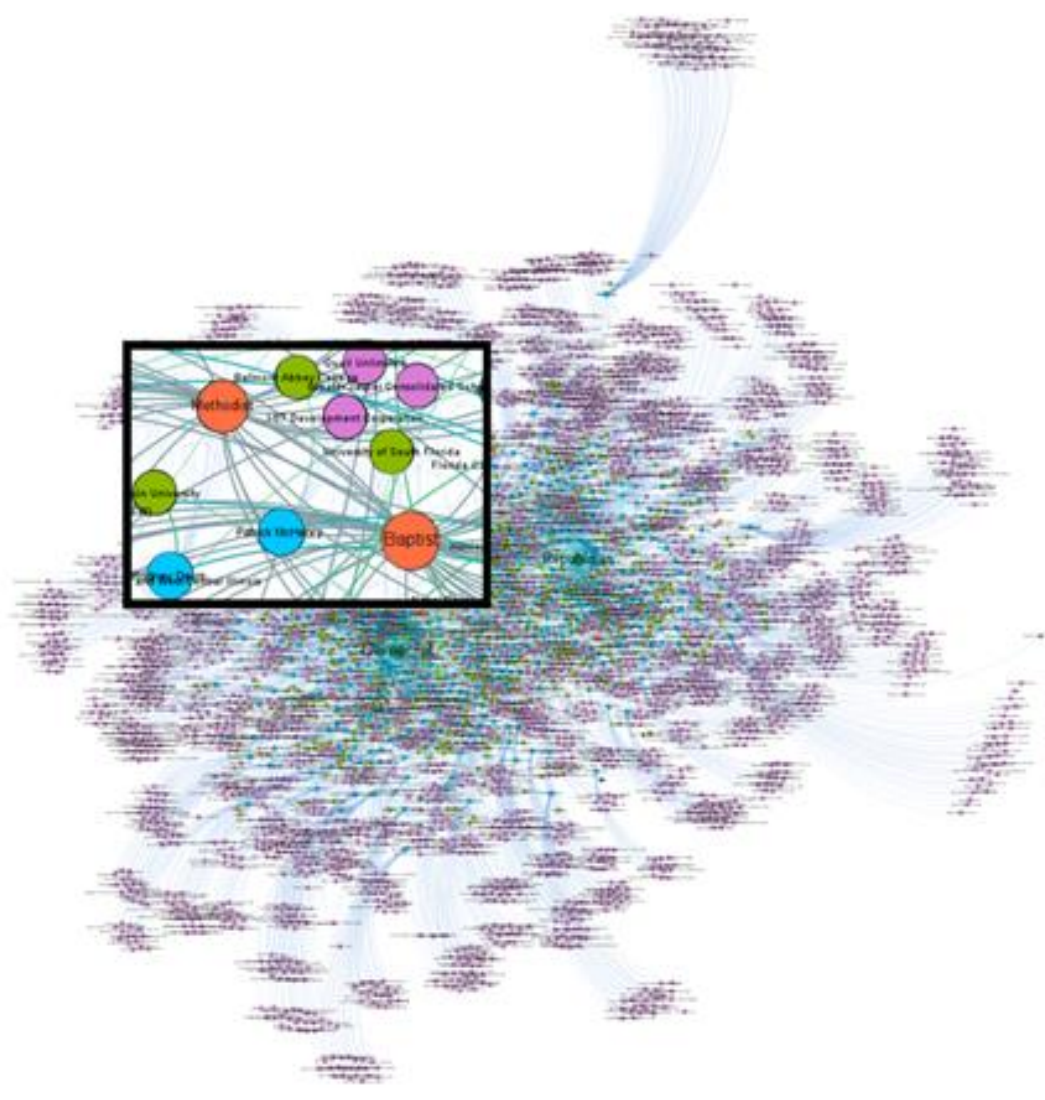

Fig. 3. Graph

In the figure, the size of a vertex is directly related to the number of edges adjacent to it, and the thickness of an edge is directly related to its weight. The following colors are used in the figure:

- $\quad$ orange - religion;

- $\quad$ blue-green - party;

- $\quad$ green - educational institution;

- $\quad$ blue - politician;

- purple - organization.

Due to the layout method, the graphs were divided into two clusters: Democrats and Republicans, but due to the large number of nodes, the data cannot be analyzed in detail.

Filters can be used to refine the information. For example, the following fragment of the graph shows which university representatives of the states most often graduate from (see Fig. 4).

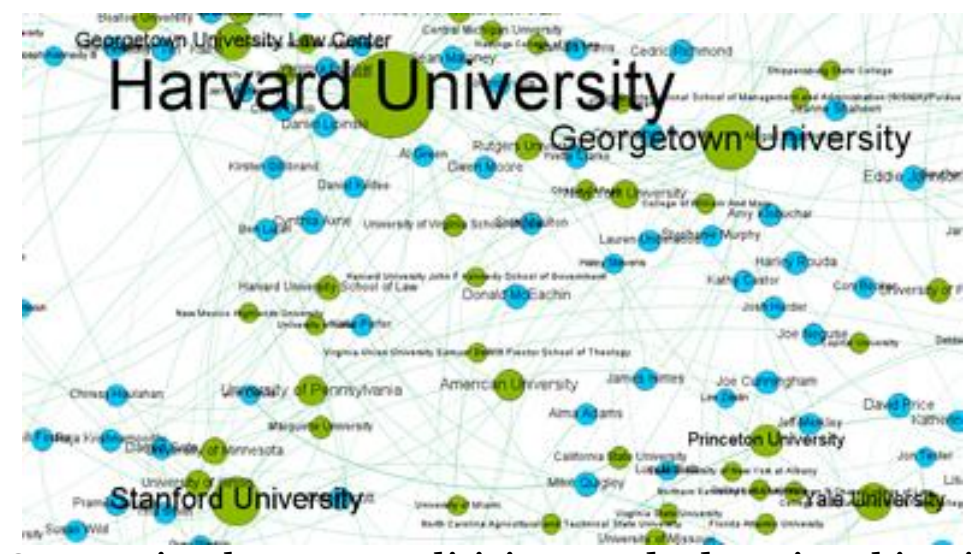

Fig. 4. Connection between politicians and educational institutions 
Harward, Georgetown and Stanford are seen to be the leading educational institution on the number of graduates.

We can also view information about the relationship of an individual object or group of objects. To do this we build a new graph (see Fig. 5), leaving the politicians of the states of Texas, California and Florida in it. The choice of states is due to the fact that they have more representatives than other states. In the new graph, the color scheme has been changed, a division by color between states has been added: representatives of Texas are highlighted in green, Florida - in red, California - in blue.

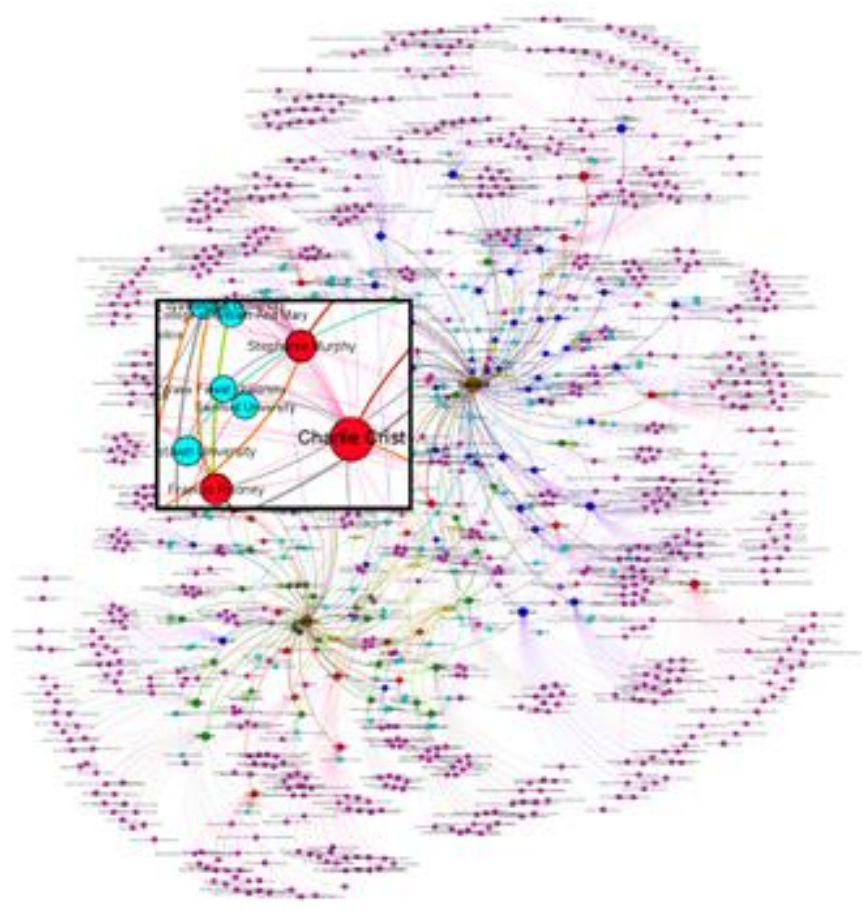

Fig. 5. Graph with representatives of three states

We check if representatives of different states can coexist with each other. To do this, we consider the subgraph, which we get after filtering of the original graph by area (see Fig. 6).

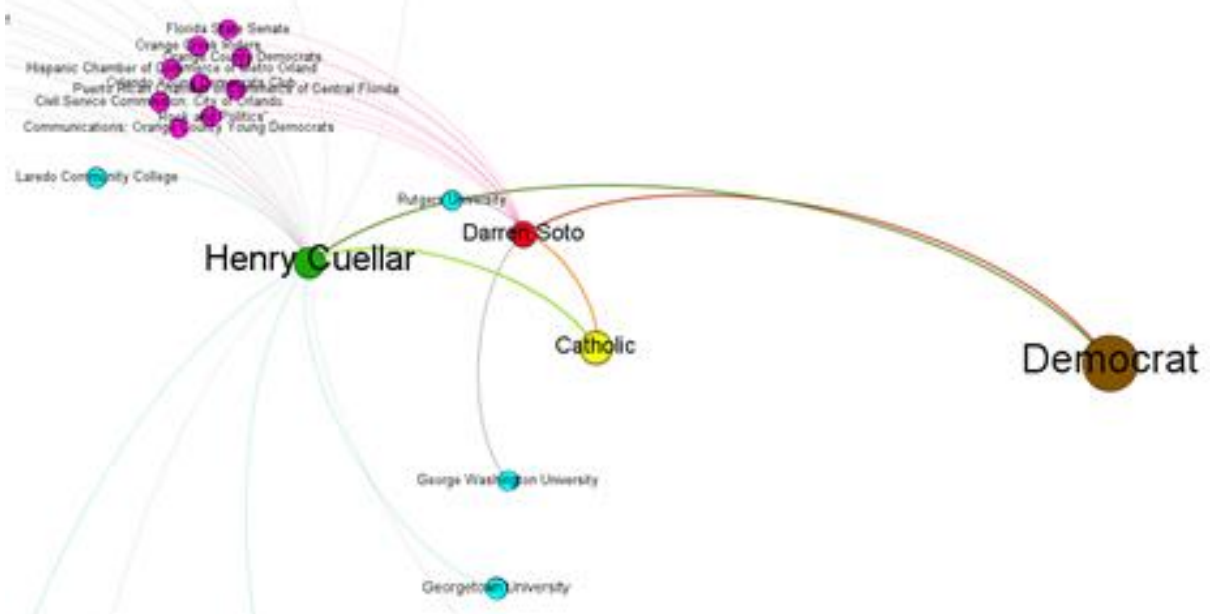

Fig. 6. Subgraph after filtering

The figure shows that Henry Cuellar, a representative from Texas, and Darren Soto, a representative from Florida, are close to each other and it can be assumed that their views are 
similar. As we can see, despite the fact that they are from different states, they have relationships with the same organizations. Both are Catholic, and belong to the Democratic Party. It tells us that the chosen Force Atlas layout method confirms the main hypothesis.

Generally speaking, it is impossible to ensure that these representatives will be close to each other if we distribute the weights of the edges in a different way. For example, remove the normalization of the edges of the form "person-educational institution" or make some type of relationship less significant. Ultimately, the constructed graph will largely depend on how the edges are weighted and how the graph is laid. This, in turn, will affect what conclusions will be drawn from the results of the graph analysis.

\section{Conclusion}

The task of visualizing linked multidimensional objects is relevant in the field of data analysis. The described technology offers a working approach to constructing graphs for an object such as a 'politician'.

Isolation of internal entities of various nature from a complex object allows you to build relationships between these entities and draw certain conclusions about them. In particular, the most graduating educational institution is Harvard University. The construction of several graphs allows us to analyze in more detail and find internal relationships - for this, Gephi provides a wide range of filtering functionality.

The existing graph can be supplemented for completeness. For example, you can add the node of the 'state' type and check if representatives of one state are located around such node. It will confirm or refute the main hypothesis that objects close in a multidimensional volume of data should be located close to each other on the graph. Any such addition increases the number of relationships and complicates visual perception, but allows you to identify additional relationships.

An alternative way to analyze related objects is to use a graph database. For example, using neo4j database it becomes possible to conduct queries that cannot be implemented using Gephi.

In future it is planned to consider the graph in respect to solving practical problems, whether it be an analysis of educational institutions or lobbying organizations, or religions depending on the states, etc.

The use of such graph models seems extremely promising from the point of view of analyzing a large amount of information not only on such a complex object as a person, but also on organization technology, etc.

\section{Acknowledgements}

The study was carried out at the expense of the Russian Science Foundation grant (project No. 19-71-30008).

\section{References}

1. Kulik, S., Shtanko, A.: Using convolutional neural networks for recognition of objects varied in appearance in computer vision for intellectual robots. Procedia Computer Science 169, 164-167 (2020).

2. Onykiy, B., Artamonov, A.A., Tretyakov, E.S. Ionkina, K.V.: Visualization of large samples of unstructured information on the basis of specialized thesauruses. Scientific Visualization 9(5), 54-58 (2017).

3. Tretyakov, E.S., Tukumbetova, R.R., Artamonov, A.A.: Methodology of Analysis of Similar Objects with the Use of Modern Visualization Tools. Mechanisms and Machine Science 80, 113-119 (2020).

4. Artamonov, A.A., Leonov, D.V., Nikolaev, V.S., Onykiy, B.N., Pronicheva, L.V., Sokolina, K.A., Ushmarov, I.A.: Visualization of semantic relations in multi-agent systems. Scientific Visualization, 6 (3), 68-76 (2014). 
5. Zinoviev, A. Y. Visualization of multidimensional data. - Krasnoyarsk State Technical University, 2000. - $180 \mathrm{p}$.

6. Bondatev A.E., Bondarenko A.V., Galaktionov V.A., Klyshinsky E.S. Visual analysis of clusters for a multidimensional textual dataset /Scientific visualization, T.8, № 3, 2016, pp. 1-24.

7. Kulik, S.D., Shtanko, A.N.: Experiments with Neural Net Object Detection System YOLO on Small Training Datasets for Intelligent Robotics. Mechanisms and Machine Science 80, 157-162 (2020).

8. Kulik, S.D.: Neural network model of artificial intelligence for handwriting recognition. Journal of Theoretical and Applied Information Technology 73(2), 202-211 (2015).

9. Votesmart, https://justfacts.votesmart.org, last accessed 2020/06/10.

10. Govtrack, https://www.govtrack.us, last accessed 2020/06/10.

11. Grigorieva, M.A., Aulov, V.A., Golosova, M.V., Gubin, M.Y., Klimentov, A.A.: Data knowledge base prototype for modern scientific collaborations. In: Selected Papers of the 7th International Conference Distributed Computing and Grid-technologies in Science and Education, pp. 26-33. CEUR, Dubna (2016).

12. Galkin, T., Popov, D., Pilyugin, V., Grigorieva, M.: The visualization method pipeline for the application to dynamic data analysis. In: Proceedings of the 27th Symposium on Nuclear Electronics and Computing, pp. 295-299. CEUR, Budva (2019).

13. Galkin, T.P., Grigorieva, M.A., Klimentov, A.A., Korchuganova, T.A., Milman, I.E., Pilyugin, V.V., Titov, M.A.: Visual cluster analysis for computing tasks at workflow management system of the ATLAS experiment at the LHC. In: GraphiCon 2018 - 28th Interna-tional Conference on Computer Graphics and Vision, pp. 111-114. GraphiCon Scientific Society, Tomsk (2018).

14. Gephi, https://gephi.org, last accessed 2020/06/28. 